\title{
基于全寿命周期的钢管混凝士结构分析理论及其应用
}

\author{
韩林海 $^{1^{*}}$, 杨有福 ${ }^{2}$, 杨华 ${ }^{3}$, 李威 ${ }^{1}$ \\ 1. 清华大学土木工程系, 北京 100084 ; \\ 2. 大连理工大学海岸和近海工程国家重点实验室, 大连 116024; \\ 3. 哈尔滨工业大学土木工程学院, 哈尔滨 150090 \\ * 联系人, E-mail: 1hhan@tsinghua.edu.cn
}

2020-05-21 收稿, 2020-07-08 修回, 2020-07-10 接受, 2020-07-13 网络版发表 国家自然科学基金重点项目(51838008)资助

摘要钢管混凝土是指在钢管中填充混凝土而成, 且钢管及其核心混凝土能共同承受外荷载作用的结构形式. 组 成该类结构的材料间协同互补作用，可有效避免或延缓钢管发生局部屈曲及混凝土发生脆性破坏。钢管混凝土具 有强度高、塑性和韧性好、抗震和抗火性能优越、施工方便等一系列技术优势, 已在工业厂房、地铁站、输电杆 塔、高层建筑以及桥梁等现代工程结构中得到广泛应用, 取得了良好的建筑效果和经济效益。本文简要回顾了钢 管混凝土结构的发展概况; 针对全寿命周期服役过程中长期荷载和复杂受力两种常遇作用、地震和火灾两种极端 作用工况, 阐述了基于全寿命周期的钢管混凝土结构分析理论及其应用; 最后, 对钢管混凝土结构研究的发展趋势 进行了简要展望。

关键词钢管混凝土结构, 全寿命周期, 约束效应, 约束效应系数, 本构模型, 分析理论

钢管混凝土是指在钢管中填充混凝土而成，且钢 管及其核心混凝土能共同承受外荷载作用的结构形式. 与传统的钢结构和钢筋混凝土结构相比，钢管混凝土 是一种相对新型的结构形式. 钢管混凝土工作的基本 原理是: 受力过程中, 钢和混凝土两种材料共同工作并 协同互补, 即钢管对核心混凝土产生约束, 使其处于复 杂应力状态，从而提高其抗压强度，延性也随之改善. 同时, 混凝土对钢管产生支撑作用, 延缓或避免受压钢 管发生局部屈曲, 保证钢材强度得以充分发挥 ${ }^{[1]}$. 圆形 和方、矩形是工程中广泛应用的钢管混凝土 (concretefilled steel tubes, CFST)截面形式 ${ }^{[1 \sim 5]}$. 图1以圆形截面钢 管混凝土轴心受压短构件为例，给出了钢管及其核心 混凝土间的相互作用示意图，其中 $p$ 为钢管与混凝土间 的相互作用应力.

本文拟简要回顾钢管混凝土结构的发展概况，阐
述基于全寿命周期的钢管混凝土结构分析理论及其应 用, 并对钢管混凝土结构研究的发展趋势进行展望.

\section{1 钢管混凝士结构的发展概况}

钢管混凝土结构的发展经历了逐渐演变的过程. 19世纪70年代，英国赛文铁路桥(Severn Railway Bridge) 采用了钢管混凝土桥墩, 是采用钢管混凝土形 式的较早报道之一. 早期钢管混凝土的外钢管一般为 无缝管或普通强度的热轧管和铸管等, 核心混凝土通 常为普通强度混凝土 ${ }^{[6,7]}$. 自 20 世纪60年代开始, 学者 们对钢管混凝土压弯构件的力学性能、钢管与核心混 凝土界面的粘接性能等进行了研究, 且以圆形截面构 件为主.

20世纪70 80年代开始，钢管混凝土结构研究由基 本静力性能逐步延伸至抗震性能、抗火性能及长期荷 


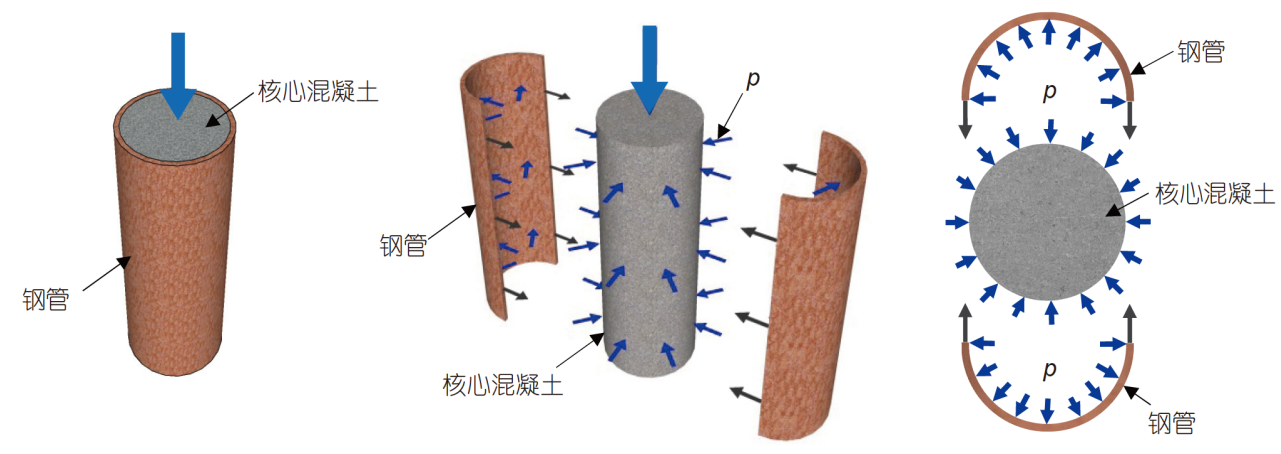

图 1 (网络版彩色)轴压荷载作用下圆钢管混凝土的钢管及其核心混凝土间的相互作用

Figure 1 (Color online) Interaction between steel tube and its core concrete of circular CFST under axial compression

载作用下的性能等. 20世纪90年代起, 钢管混凝土的工 程应用有了长足进展，机械性能相对更好的冷弯钢管 与焊接钢管等被广泛应用于钢管混凝土结构中 ${ }^{[2,3,8]}$, 使 外钢管的进一步薄壁化和尺寸多样化成为可能; 高强 混凝土(如强度等级高于 $\mathrm{C} 60 \sim \mathrm{C} 80$ 的混凝土)的采用, 使 钢管混凝土进一步高强化, 构件截面更小, 经济性能更 好. 在这一阶段, 为适应建筑构造的要求, 方、矩形截 面钢管混凝土在工程中的应用逐渐增多. 在力学性能 研究方面, 学者们将单一静载下的受力性能研究进一 步扩展至压弯剪和压弯扭等复合受力性能，抗震性能 研究也进一步深人. 同时, 方、矩形截面钢管混凝土设 计方法的研究取得了较大进展，对采用薄壁钢管的钢 管混凝土也有不少研究报道 ${ }^{[1,9]}$.

在上述研究工作的基础上, 国内外已制定了多部 钢管混凝土结构相关的技术标准. 如我国国家标准 《钢管混凝土拱桥技术规范》GB $50923^{[10]}$ 和《钢管混 凝土结构技术规范》 GB $50936^{[11]}$ 、中国工程建设标准 化协会标准《钢管混凝土结构技术规程》 CECS $28^{[12]}$ 和《矩形钢管混凝土结构技术规程》 CECS $159^{[13]}$ 、 电力行业标准《钢-混凝土组合结构设计规程》 DL/T $5085^{[14]}$ 和福建省工程建设标准《钢管混凝土结构技术 规程》DBJ/T13-51 ${ }^{[15]}$ 等, 日本Recommendations for Design and Construction of Concrete Filled Steel Tubular Structures $^{[16]}$ 、美国Specification for Structural Steel Buildings ANSI/AISC 360 $0^{[17]}$ 和欧洲Design of Composite Steel and Concrete Structures EN 1994-1-1 ${ }^{[18]}$ 等. 上述工 程建设标准给出了钢管混凝土结构在材料、构件、节 点和施工等方面的相关设计条文，推动了该类结构在 基础工程、工业厂房、地铁站、高层建筑及桥梁等中 的应用.
近 20 年来，钢管混凝土结构的研究呈现出一些新 动向: 一方面, 在传统钢管混凝土结构的基础上, 基于 全寿命周期的钢管混凝土分析理论得到深人发展; 另 一方面, 为了进一步提高结构的性能, 采用新截面(椭 圆形和多边形等)、新材料(如高强高性能混凝土, 屈服 强度 $\geqslant 460 \mathrm{MPa}$ 的高强钢材、不锈钢等) 和新构成形式 (如中空夹层钢管混凝土等)的钢管混凝土结构 ${ }^{[1,4,19 \sim 22]}$ 陆续出现. 此外, 还进一步拓展为由钢管混凝土构件和 传统钢或混凝土构(部)件通过优化混合而成的高性能

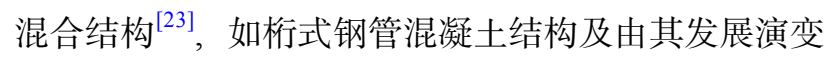
而成的钢管混凝土加劲混合结构等. 上述发展动向的 归纳如图2所示.

\section{2 基于全寿命周期的钢管混凝土结构分析 理论}

众所周知，工程结构的全寿命周期包括设计、施 工、运营和维护等环节. 在全寿命周期内, 工程结构的 可靠性将影响可持续城镇化建设过程中的环境、材

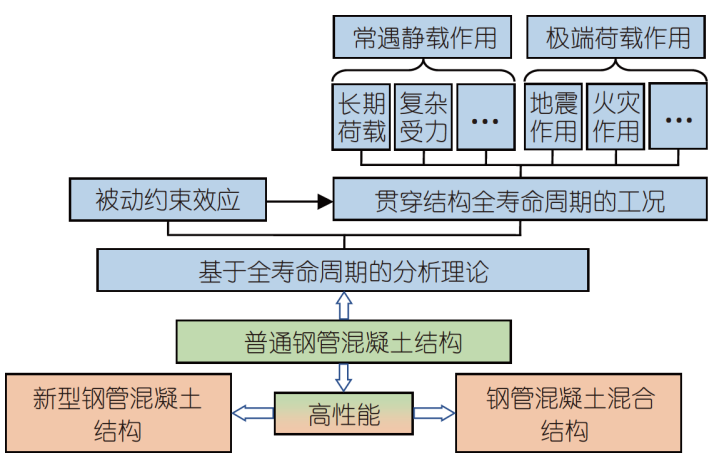

图 2 (网络版彩色)钢管混凝土结构研究的发展动向

Figure 2 (Color online) Development trend of the CFST structures research 
料、信息、能源、经济等诸多方面.

近年来，钢管混凝土结构的应用领域和规模进一 步扩大, 已由在工业厂房、地铁站、高层建筑和拱桥 等工程的应用, 进一步拓展至高䇯电视塔、大跨度桥 梁、大跨越输电塔、高烈度区大跨、重载结构中的多 种主体受力结构, 工程难度前所未有, 所处环境与所需 考虑的作用形式(如腐蚀、爆炸、冲击等)趋于复杂, 分 析与设计的精细化需求进一步提升. 因此, 很难也不应 将钢管混凝土全寿命周期内的设计、施工、维护等环 节割裂开来. 这些环节中提取出的荷载、温度、冲击 能、腐蚀等作用, 将影响或耦合影响钢管混凝土结构 的可靠性. 为此，只有解决上述环节中的基础理论问 题，系统建立基于全寿命周期的钢管混凝土结构分析 理论才能成为可能, 同时, 才能适用于现在已经出现 的, 以及未来在此基础上进一步发展的新型钢管混凝 土组合结构与混合结构.

在全寿命周期的维度上, 钢管混凝土结构分析理 论特色总体上体现在如下 3 个方面 ${ }^{[1]}$ : (1) 综合考虑施 工因素(如钢管制作、核心混凝土浇灌等)、长期荷载 (如混凝土收缩和徐变)及环境作用影响(如氯离子腐蚀 等)的钢管混凝土结构分析理论; (2) 全寿命周期服役过 程中, 钢管混凝土结构在可能导致其破坏的极端作用 下(如强烈地震、火灾和撞击等)力学性能的分析理论, 以及考虑多种作用相互耦合的分析理论与方法; (3) 基 于全寿命周期的钢管混凝土结构设计原理与方法.

围绕上述 3 个方面，作者们基于系统的精细化实 验、模拟和工程实践, 攻克了核心混凝土受被动约束 效应机制难题, 建立了其通用型本构模型; 揭示了全寿 命周期服役过程中的长期荷载和复杂受力两种常遇作 用下、地震和火灾两种极端作用下结构的损伤机理、 分析和计算方法，为钢管混凝土结构安全、合理的推 广应用提供了理论基础.

\section{1 被动约束效应概念}

钢管混凝土性能优势的本源在于受力过程中钢管 对其核心混凝土的被动约束效应，这一效应使其在工 作机制上具有极大的复杂性. 如何在概念上准确揭示 约束效应机制, 量化约束效应幅值, 进而提出其核心混 凝土本构模型是该领域的基础性问题.

以往，有学者“借用”传统筂筋约束混凝土的方法, 采用“套䈨指标”这一参数进行钢管混凝土构件承载力 计算, 也有学者采用钢管的径(宽)厚比作为基本参数衡
量约束效应的变化. 这些方法都不能完全满足基于全 寿命周期的钢管混凝土结构分析理论研究，这是因为: (1) 钢管混凝土在受力过程中, 钢管一般处于三向应力 状态且存在发生局部屈曲的趋势, 而䈐筋约束混凝土 中的筷筋处于环向受拉的单向应力状态; (2) 钢管混凝 土的基本性能不仅决定于钢管和混凝土本身的几何尺 寸与物理特性参数, 也决定于二者的“匹配”关系, 仅采 用钢管径(宽)厚比作为基本参数难以准确描述被动约 束效应; (3) 尚需准确反映全寿命周期服役过程中，长 期应力、循环应力和高温作用下约束效应的变化 规律.

基于上述考虑，提出了“约束效应系数”概念，并确 定用约束效应系数 $(\xi)$ 作为衡量全寿命周期服役过程中 钢管对其核心混凝土被动约束效应的基本参数 ${ }^{[1,24]}$. $\xi$ 的表达式如下:

$\xi=\frac{A_{\mathrm{s}} f_{\mathrm{y}}}{A_{\mathrm{c}} f_{\mathrm{ck}}}=\alpha \frac{f_{\mathrm{y}}}{f_{\mathrm{ck}}}$,

式中, $A_{\mathrm{s}}$ 和 $A_{\mathrm{c}}$ 分别为钢管和核心混凝土的横截面面积; $\alpha\left(=A_{\mathrm{s}} / A_{\mathrm{c}}\right)$ 为钢管混凝土截面含钢率; $f_{\mathrm{y}}$ 为钢材屈服强 度, 对于单一荷载与复杂受力、长期荷载及地震作用 工况，取为常温值，对于火灾下与火灾后作用工况，需 分别考虑实时温度与经历的最高温度的影响; $f_{\mathrm{ck}}$ 为核 心混凝土轴心抗压强度标准值.

上述适用于多种截面形式钢管混凝土的约束效应 系数概念，较为准确地揭示了贯穿全寿命周期的长期 荷载、复杂受力、地震和火灾工况下钢管与核心混凝 土间作用机制的物理本质，合理量化了钢管混凝土几 何尺寸与物理特性的匹配关系，即截面尺寸相同条件 下, 钢管与混凝土的材料强度比决定约束效应; 材料强 度不变情况下, 含钢率决定约束效应. 约束效应系数是 描述核心混凝土本构模型的关键参数，为新型钢管混 凝土结构和钢管混凝土混合结构分析理论的科学建立 及深人研究创造了条件. 约束效应系数还可为钢管混 凝土结构概念设计提供依据，如确定截面几何特性(截 面尺寸、含钢率)、物理参数(材料强度)及二者之间的 匹配关系优化, 以及结构抗震设计中的延性计算等.

\section{2 通用型核心混凝土本构模型}

以往, 对钢材本构模型已有系统研究, 但尚缺乏准 确、通用的核心混凝土本构模型. 以提出的约束效应 系数为基本参数, 针对全寿命周期服役过程中钢管混 
凝土结构可能承受的多种工况，作者们提出了适用于 纤维模型法和有限元法的核心混凝土受压应力-应变 关系模型. 以圆钢管混凝土为例, 式(2a)和(2b)分别给出 了短期静力荷载作用下，适用于纤维模型法和有限元 法的核心混凝土受压应力 $(\sigma)$-应变 $(\varepsilon)$ 关系模型 ${ }^{[1,20,25]}$.

(1) 纤维模型法:

$y=\left\{\begin{array}{lll}2 x-x^{2}, & (x \leq 1), \\ 1+q\left(x^{0.1 \xi}-1\right), & (\xi \geq 1.12), & (x>1), \\ x /\left[\beta_{1}(x-1)^{2}+x\right], & (\xi<1.12),\end{array}\right.$

(2) 有限元法:

$y= \begin{cases}2 x-x^{2}, & (x \leq 1), \\ x /\left[\beta_{2}(x-1)^{2}+x\right], & (x>1),\end{cases}$

式中, $x=\varepsilon / \varepsilon_{0}, y=\sigma / \sigma_{0}, \sigma_{0}$ 为峰值应力, $\varepsilon_{0}$ 为与 $\sigma_{0}$ 对应 的应变; $q 、 \beta_{1}$ 和 $\beta_{2}$ 为中间变量. 公式的适用范围是: Q235 Q460钢; C 30 C 80混凝土; 对于圆钢管混凝土, $\alpha=0.06 \sim 0.2, \xi=0.6 \sim 4.0$; 对于方、矩形钢管混凝土, $\alpha=0.1 \sim 0.2, \xi=1.0 \sim 4.0$.

在式(2)的基础上, 基于系统的试验研究, 考虑核心 混凝土徐变系数和收缩应变对与峰值应力对应应变的 影响规律, 建立了长期应力作用下核心混凝土受压应 力-应变关系模型. 以短期静力荷载作用下核心混凝土 受压应力-应变关系模型为骨架曲线, 考虑循环应力下 的卸载刚度退化和软化特性, 建立了循环应力作用下 核心混凝土应力-应变关系模型. 考虑全过程火灾作用 下钢管混凝土经历常温、高温、降温和高温后的各个 阶段, 基于温度对被动约束效应影响规律确定了高温 下(后)约束效应系数计算方法, 同时考虑温度对核心混 凝土峰值应力及与之对应应变的影响, 建立了能描述 在这一复杂温度变化路径下的核心混凝土受压应力应变关系模型 ${ }^{[1]}$.

图3所示为不同约束情况下核心混凝土受压应力应变关系模型示意图，其中 $\sigma_{02}$ 和 $\sigma_{01}$ 分别为对应强、弱 约束情况下的峰值应力, $\varepsilon_{02}$ 和 $\varepsilon_{01}$ 分别为对应的应变. 约 束效应系数 越大, 受力过程中钢管对核心混凝土的约 束作用越强, 核心混凝土峰值应力及与之对应应变的 提高幅度以及应变软化的降低幅度越大.

由此, 作者们基于约束效应系数, 建立了核心混凝 土通用型本构模型, 为在钢管混凝土构件与结构层次 上建立精细化数值模拟模型，厘清贯穿全寿命周期的

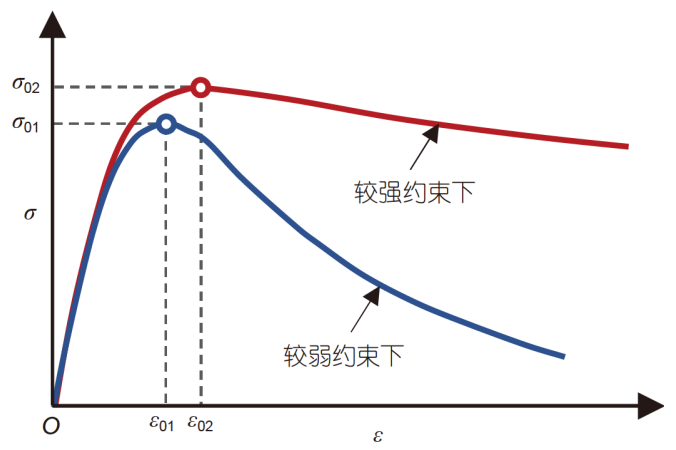

图 3 (网络版彩色) 不同约束情况下核心混凝土受压应力-应变关系 示意图

Figure 3 (Color online) Schematic diagram of compressive stressstrain relationship of core concrete under different confinements

长期荷载、复杂受力、地震和火灾工况下结构的损 伤机制, 进一步建立相应的承载力计算模型创造了 条件.

\section{3 多工况作用下钢管混凝土结构的计算分析方法}

如何建立常遇静载和极端荷载作用下结构的损伤 机理分析与承载力计算方法，为大型复杂钢管混凝土 工程结构安全性设计提供保障是该领域的另外一个基 础性问题.

全寿命周期服役过程中，钢管混凝土结构常处在 轴压(拉)、压(拉)弯、压扭、压弯扭、压弯剪甚至压 弯扭剪等单一或复杂受力状态(如图4(a)所示, 图中 $N$ 、 $M 、 T$ 和 $V$ 分别代表轴力、弯矩、扭矩和剪力), 为此, 建 立了钢管混凝土构件在压(拉)、弯、扭、剪及其复合 受力状态下力学行为分析的精细化有限元模型. 对轴 心受压(拉)、受弯、压(拉)弯、受扭、受剪、压扭、 弯扭、压弯扭、压弯剪和压弯扭剪受力状态下的荷 载-变形关系曲线进行了全过程模拟和分析, 有计划地 开展了系列物理实验, 明晰了各受力阶段钢管及其核 心混凝土截面的应力状态及其相互作用规律，以及不 同加载路径下钢管混凝土构件的力学特性, 揭示了复 杂受力状态下钢管混凝土构件的损伤特征和极限状态 变化规律. 在系统参数分析的基础上, 提出了相应的承 载力计算方法 ${ }^{[1,20,26,27]}$, 如图4(b)所示, 图中 $N_{\mathrm{u}} 、 M_{\mathrm{u}} 、 T_{\mathrm{u}}$ 和 $V_{\mathrm{u}}$ 分别代表轴压(拉)、受弯、受扭和受剪承载力.

针对全寿命周期服役过程中的钢管混凝土结构可 能遭受的地震作用，建立了低周反复荷载作用下钢管 混凝土构件力学性能分析的精细化有限元模型, 揭示 了不同截面形状钢管混凝土构件的弯矩-曲率、水平 

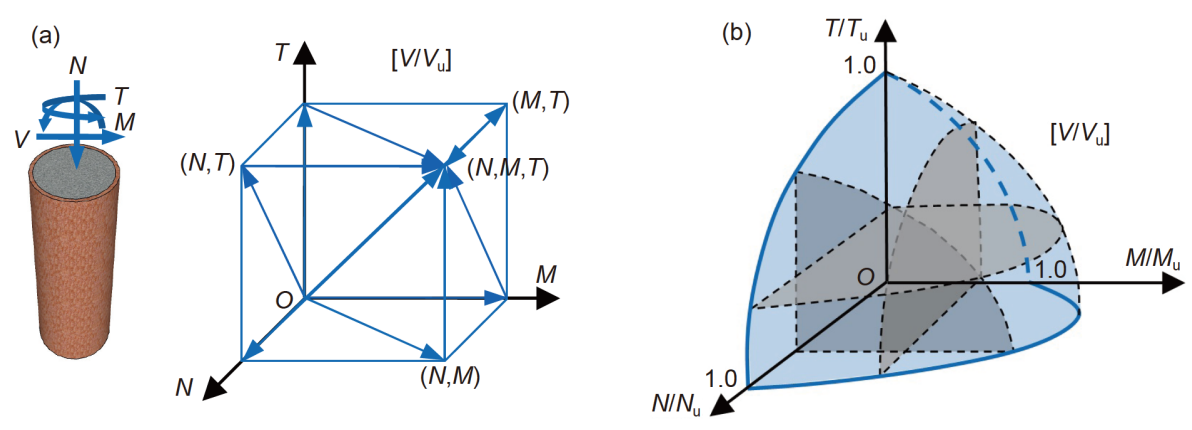

图 4 (网络版彩色)复杂受力钢管混凝土构件. (a) 加载路径; (b) 承载力包络面

Figure 4 (Color online) CFST members under complex loading. (a) Loading paths; (b) envelope surface for bearing capacity

荷载-水平位移滞回关系特性及其损伤规律，提出了钢 管混凝土构件荷载-位移滞回关系模型(如图5所示，图 中 $A\left(A^{\prime}\right) 、 B\left(B^{\prime}\right)$ 和 $C\left(C^{\prime}\right)$ 代表骨架曲线控制点)以及位移 延性系数计算的数学模型 ${ }^{[1,4,28]}$.

科学进行钢管混凝土构件的抗火设计与火灾后评 估, 需要考虑包括升温和降温在内的火灾作用全过程. 在这一过程中, 钢管混凝土同时承受荷载与温度的复 杂时变作用. 若将作用路径进行简单分离, 即采用图6 (a)中所示的路径 $\mathrm{AC}^{\prime} \mathrm{D}^{\prime} \mathrm{E}^{\prime}$, 无法准确模拟火灾作用全过 程中的应力重分布和累积损伤，进而高估其抗火性能. 为此, 作者们采用了可较为真实反映火灾作用全过程 的路径ABCDE，建立了综合考虑荷载、温度和时间耦 合作用下的钢管混凝土构件荷载-变形关系曲线及耐 火极限的理论模型(如图6(b)所示, 图中 $T$ 代表温度, $\varepsilon_{\sigma}(\sigma, T) 、 \varepsilon_{\mathrm{th}}(T) 、 \varepsilon_{\mathrm{c}, \mathrm{tr}}(\sigma, T)$ 分别代表应力应变、热膨胀 应变、瞬态热应变，“+”和“一”分别代表拉应变和压应 变), 明晰了全过程火灾作用下钢管混凝土构件的典型 破坏特征与荷载-变形全过程关系曲线的变化规律, 分 析了关键参数对钢管混凝土柱耐火极限、典型破坏特 征及其承载能力的影响规律，提出了火灾作用下钢管

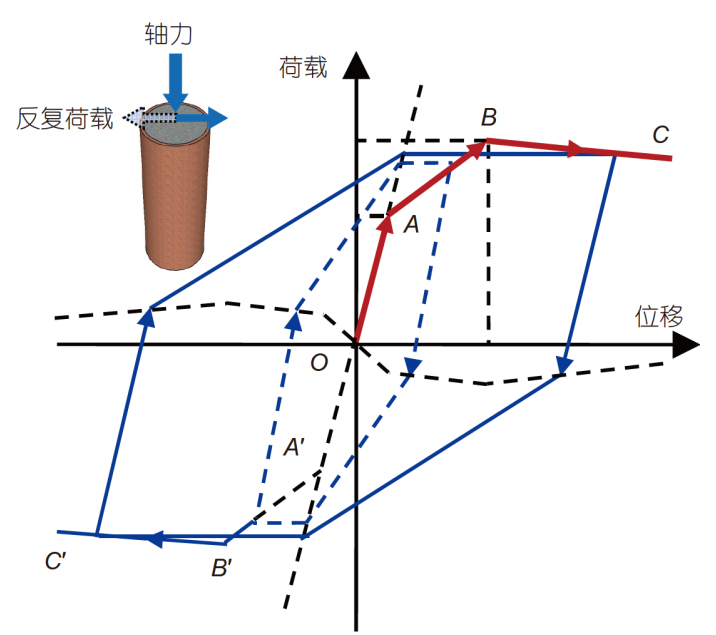

图 5 (网络版彩色)钢管混凝土构件的荷载-位移滞回关系模型 Figure 5 (Color online) Load-displacement hysteretic model of CFST members

混凝土受压构件的承载力和防火保护设计方法，建立 了火灾作用后构件承载力和变形计算的数学模型以及 压弯构件的恢复力模型，实现了综合考虑服役期间受 力与受火全过程影响的钢管混凝土构件损伤评 估 ${ }^{[1,25,29]}$.

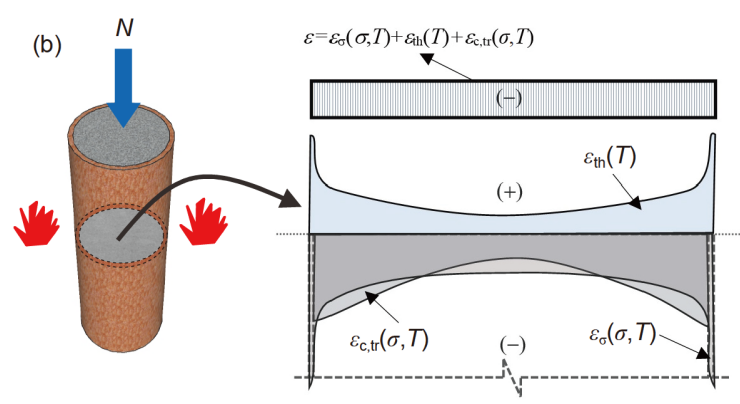

图 6 (网络版彩色)全过程火灾作用下的钢管混凝土构件. (a) 荷载-温度-时间路径; (b) 理论模型

Figure 6 (Color online) CFST members under full-range fire. (a) Load-temperature-time paths; (b) theoretical model 


\section{3 基于全寿命周期的钢管混凝士结构分析理 论的应用}

基于全寿命周期的钢管混凝土结构分析理论的建 立为普通钢管混凝土结构的理论研究、计算与设计奠 定了基础. 该理论成果也被拓展应用到一些新型钢管 混凝土构件及钢管混凝土框架结构等的理论研究, 并 被应用于大型复杂钢管混凝土工程结构的设计中; 部 分成果还被多部工程技术标准采纳.

\section{1 新型组合结构和混合结构工作机理研究}

基于全寿命周期的钢管混凝土结构分析理论在新 型钢管混凝土构件、钢管混凝土柱-钢(钢筋混凝土、 组合)梁连接节点、钢板组合剪力墙、钢管混凝土柱钢(型钢混凝土)梁平面框架和钢管混凝土框架结构的 研究中得到应用, 体现出较强的通用性.

( i ) 新型钢管混凝土构件. 约束效应系数(式(1))的 概念被拓展应用于新型钢管混凝土, 以研究不同材料 或组成部分之间的相互作用，如椭圆形钢管混凝 土 $^{[19]}$ 、中空夹层钢管混凝土 ${ }^{[22]}$ 、内置FRP(fibre reinforced polymer/plastics)管钢管混凝土 ${ }^{[30]}$ 、带加劲肋钢 管混凝土 ${ }^{[31]} 、 内$ 置型钢钢管混凝土 ${ }^{[32]}$ 、 L 形钢管混凝 土 $^{[33]}$ 、预制装配式钢管混凝土 ${ }^{[34]}$ ，以及新型管材 (如不 锈钢管、高强钢管)和/或新型内填混凝土材料(高强混 凝土、海水海砂混凝土)构成的钢管混凝土 ${ }^{[35 \sim 38]}$.
式(2)所示的核心混凝土本构模型被拓展用于中空 夹层钢管混凝土柱 ${ }^{[22]}$ 、内置FRP管钢管混凝土柱 ${ }^{[30]}$ 、 带加劲肋钢管混凝土柱 ${ }^{[31]}$ 、内置型钢钢管混凝土 ${ }^{[32] 、}$ 不锈钢管混凝土柱 ${ }^{[36]}$ 、螺旋焊钢管混凝土柱 ${ }^{[39]}$ 、哑铃 型钢管混凝土构件 ${ }^{[40]}$ 、FRP约束钢管混凝土柱 ${ }^{[41]}$ 、中 空型外壁钢板-混凝土组合桥塔塔柱 ${ }^{[42]}$ 、五边形钢管 混凝土翼缘组合梁 ${ }^{[43]}$ 等的静力性能，不锈钢管混凝土 柱 ${ }^{[44]}$ 和部分填充混凝土矩形钢管混凝土柱 ${ }^{[45]}$ 的抗冲击 性能, 以及L形钢管混凝土混合构件 ${ }^{[33]}$ 、预制装配式钢 管混凝土柱 ${ }^{[34]}$ 等的抗震性能研究. 钢管混凝土构件承 载力计算方法被拓展用于内置FRP管钢管混凝土柱 ${ }^{[30]}$ 和钢管海水海砂混凝土柱 ${ }^{[37]}$ 的承载力计算方法的提 出. 图7所示为部分新型钢管混凝土构件示意图.

(ii) 钢管混凝土柱-钢(钢筋混凝土、组合)梁连接 节点. 式(2)所示的核心混凝土本构模型被拓展应用于 方钢管混凝土柱-钢梁单边螺栓节点 ${ }^{[46]}$ 等的静力性能, 以及方钢管混凝土柱-钢梁内隔板节点 ${ }^{[47]}$ 、方钢管混 凝土柱-组合梁节点 ${ }^{[48]}$ 、方钢管混凝土柱-钢筋混凝土 梁贯通节点 ${ }^{[49]}$ 等的抗震性能研究. 图8所示为部分钢管 混凝土柱-钢(钢筋混凝土、组合)梁连接节点示意图.

(iii) 钢板组合剪力墙. 式(2)所示的核心混凝土本 构模型被拓展用于双钢板组合剪力墙 ${ }^{[00}$ 和多腔双钢板 组合剪力墙 ${ }^{[51]}$ 的抗震性能研究. 图9所示为钢板组合剪 力墙示意图.

(iv) 钢管混凝土柱-钢(型钢混凝土)梁平面框架.

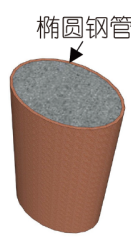

(a)

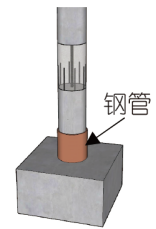

(g)

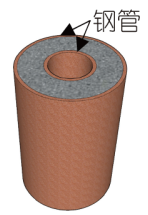

(b)

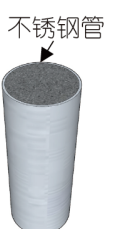

(h)

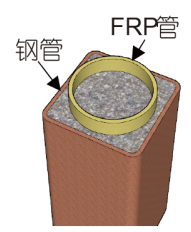

(c)

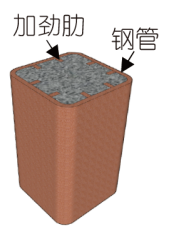

(d)

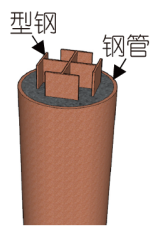

(e)

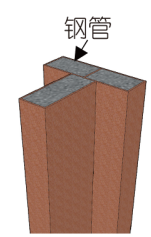

(f)

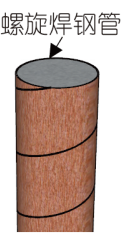

(i)

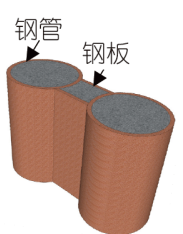

(j)

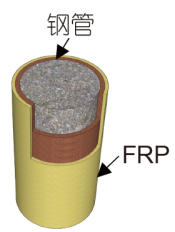

(k)

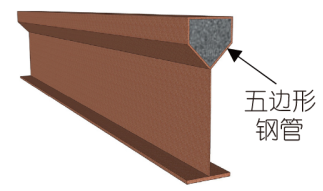

(I)

图 7 (网络版彩色)新型钢管混凝土构件. (a) 椭圆钢管混凝土柱; (b) 中空夹层钢管混凝土柱; (c) 内置FRP管钢管混凝土柱; (d) 带加劲肋钢管混 凝土柱; (e) 内置型钢管混凝土柱; (f) T形钢管混凝土柱; (g) 预制装配式钢管混凝土柱; (h) 不锈钢管混凝土柱; (i) 螺旋焊钢管混凝土柱; (j) 哑铃 型钢管混凝土构件; (k) FRP约束钢管混凝土柱; (1) 五边形钢管混凝土翼缘组合梁

Figure 7 (Color online) Innovative CFST members. (a) Elliptical CFST column; (b) double-skin CFST column; (c) CFST column with inner FRP tube; (d) CFST column with stiffeners; (e) steel-reinforced CFST column; (f) T-shaped CFST column; (g) precast segmental CFST column; (h) concretefilled stainless steel tube column; (i) concrete-filled spiral welded steel tube column; (j) dumbbell-shaped CFST member; (k) FRP confined CFST column; (1) concrete-filled pentagonal flange beam 


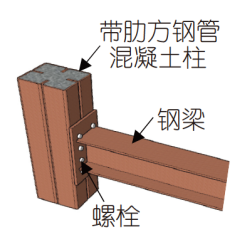

(a)

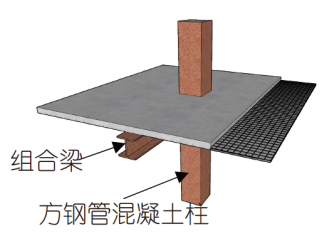

(c)

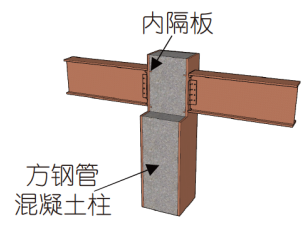

(b)

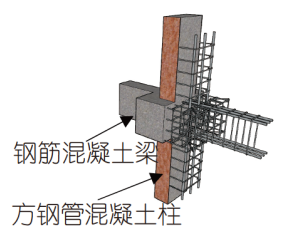

(d)
图 8 (网络版彩色) 钢管混凝土柱-钢(钢筋混凝土、组合)梁连接节 点. (a) 方钢管混凝土柱-钢梁单边螺检节点; (b) 方钢管混凝土柱-钢 梁内隔板节点; (c) 方钢管混凝土柱-组合梁节点; (d) 方钢管混凝土 柱-钢筋混凝土梁贯通节点

Figure 8 (Color online) CFST column to steel/reinforced concrete/ composite beam joints. (a) Square CFST column to steel beam blind bolted joint; (b) square CFST column to steel beam internal diaphragm joint; (c) square CFST column to composite beam joint; (d) square CFST column to reinforced concrete beam through-beam joint

式(2)所示的核心混凝土本构模型被拓展用于方钢管混 凝土柱-型钢混凝土梁框架 ${ }^{[52]}$ 、钢管混凝土加劲混合 柱-型钢混凝土梁框架 ${ }^{[33]}$ 和带支撑内置夹层板的方钢 管混凝土柱-钢梁框架 ${ }^{[54]}$ 的抗震性能, 以及钢管混凝土 柱-工字形钢梁框架 ${ }^{[55]}$ 的火灾(高温)下性能研究. 图 10 所示为部分钢管混凝土柱-钢(型钢混凝土)梁平面框架 示意图.

（V）钢管混凝土框架结构. 式(2)所示的核心混凝 土本构模型被拓展用于多层钢管混凝土框架 ${ }^{[56]}$ 和高层 钢管混凝土结构 ${ }^{[57]}$ 的抗震性能研究. 图11所示为钢管 混凝土框架结构示意图.

基于全寿命周期的钢管混凝土结构分析理论研究 方面取得的成果, 还为深人研究桁式钢管混凝土结构
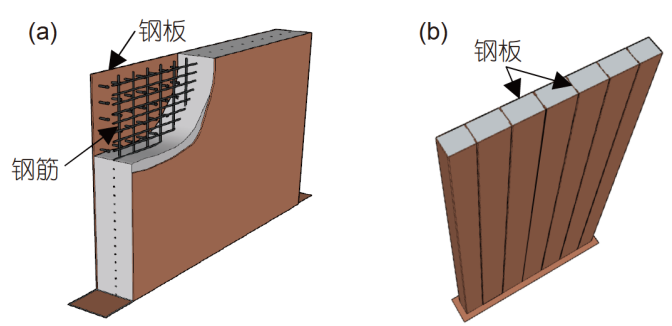

图 9 (网络版彩色)钢板组合剪力墙. (a) 双钢板组合剪力墙; (b) 多 腔钢板组合剪力墙

Figure 9 (Color online) Steel plate composite shear walls. (a) Composite shear wall with double plates and filled concrete; (b) multicell steel plate composite shear wall

和钢管混凝土加劲混合结构的工作机理和设计原理及 其在桥梁结构中的应用创造了条件 ${ }^{[23]}$.

\section{2 工程设计和工程技术标准制订}

基于全寿命周期的钢管混凝土结构分析理论的主 要成果，如核心混凝土通用型本构模型、复杂受力状 态下承载力计算方法、火灾下承载力计算和防火保护 设计方法，被应用于钢管混凝土工程结构的受力分析 与计算, 如高层/高䇯结构 ${ }^{[58 ~ 63]}$ 、大跨/空间结构 ${ }^{[64 ~ 66]}$ 及重载结构 ${ }^{[67]}$ 等.

基于全寿命周期的钢管混凝土结构分析理论的部 分成果，被多部国家标准 ${ }^{[10,68,69]}$ 、行业/团体标 准 ${ }^{[12 \sim 14,70 \sim 73]}$ 及福建省《钢管混凝土结构技术规程》

$\mathrm{DBJ} / \mathrm{T} 13-51^{[15]}$ 等工程建设标准采纳. 该理论已拓展应 用到钢管再生混凝土结构的系统研究, 并制订了该结 构的技术标准 ${ }^{[74]}$.

\section{4 结语}

钢管混凝土结构是更充分考虑结构的安全性、适 用性、和谐性以及良好的可施工性等综合因素的产物.

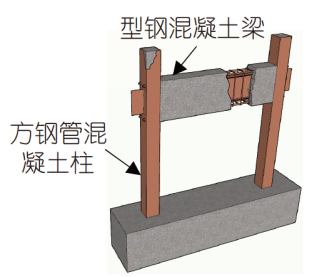

(a)

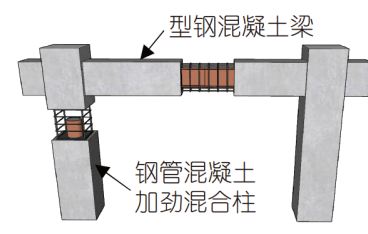

(b)

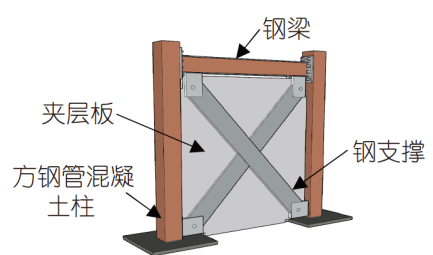

(c)

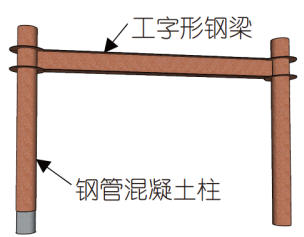

(d)

图 10 (网络版彩色)钢管混凝土柱-钢(型钢混凝土)梁平面框架. (a) 方钢管混凝土柱-型钢混凝土梁框架; (b) 钢管混凝土加劲混合柱-型钢混凝 土梁框架; (c) 带支撑内置夹层板方钢管混凝土柱-钢梁框架; (d) 钢管混凝土柱-工字形钢梁框架

Figure 10 (Color online) CFST column to steel/steel reinforced concrete beam planar frames. (a) Square CFST columns to steel reinforced concrete beam frame; (b) concrete-encased CFST column to steel reinforced concrete beam frame; (c) square CFST columns to steel beam frame with external sandwich composite wall panels; (d) CFST columns to I-shaped steel beam frame 
(a)

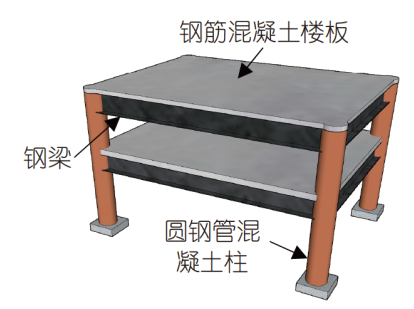

(b)

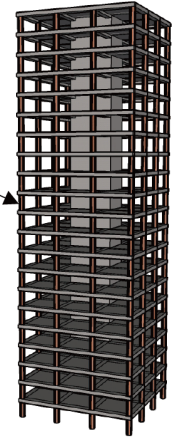

图 11 (网络版彩色)钢管混凝土框架结构. (a) 多层钢管混凝土框架; (b) 高层钢管混凝土结构

Figure 11 (Color online) CFST frame structures. (a) Multi-story CFST frame; (b) high-rise CFST structures

合理设计并应用钢管混凝土结构，符合现代工程结构 发展和建设节约型社会的需要, 有利于较大限度地实 现建设投人经济性与结构性能有效性的统一. 本文简 要回顾了钢管混凝土结构的发展概况，阐述了基于全 寿命周期的钢管混凝土结构分析理论及其应用.

目前，国内外虽然在钢管混凝土结构设计理论方 面取得了不少成果，但对钢管混凝土结构仍有不少问 题需要深人研究, 简要归纳如下:

(1) 环境友好型钢管混凝土结构设计原理研究. 在 钢管混凝土工程中研究应用高性能材料，如高强钢、 不锈钢、高韧性混凝土等, 将进一步提高材料利用率,

降低造价并提高结构的工作性能. 采用新型材料也将 给钢管混凝土结构的设计原理带来革新, 需进一步深 人开展相关研究工作.

（2）新型钢管混凝土结构及钢管混凝土混合结构 设计原理研究. 近些年, 工程技术人员在工程实践中发 展和应用了中空夹层钢管混凝土、桁式钢管混凝土结 构和钢管混凝土加劲混合结构等结构形式 ${ }^{[5,23]}$. 需在系 统研究工作机理和设计原理的基础上，制订相应的新 型钢管混凝土结构及钢管混凝土混合结构技术标准.

（3）钢管混凝土结构综合抗灾能力研究. 对现代钢 管混凝土结构抵抗灾害的能力有着更高的要求，如在 海洋环境中的大跨越输变电塔等, 要求结构更好地适 应荷载和环境长期的共同作用，满足在全寿命服役周 期内的安全性、适用性、耐久性及灾后的功能可恢复 性等要求。在极端灾害作用下以及全寿命周期内钢管 混凝土结构设计原理方面，关键基础科学问题尚有待 于继续深入研究，如爆炸、撞击以及多灾种耦合作用 下的结构破坏机制，荷载和腐蚀共同作用下的结构受 力机理等.

最后, 需要指出的是, 钢管混凝土已成为结构工程 领域的重要分支, 其内涵十分丰富, 国内外相关研究成 果众多. 对钢管混凝土结构研究和发展进行系统完整 的论述, 超出了作者们的知识和能力范围, 且已有不少 相应的文献可以参阅.

\section{参考文献}

1 Han L H. Concrete Filled Steel Tubular Structures: Theory and Practice (in Chinese). 3rd ed. Beijing: Science Press, 2016 [韩林海. 钢管混凝土结 构——理论与实践. 第三版. 北京: 科学出版社, 2016]

2 Zhong S T. Unified Theory of Concrete Filled Steel Tube: Research and Application (in Chinese). Beijing: Tsinghua University Press, 2006 [钟善 桐. 钢管混凝土统一理论——研究与应用. 北京: 清华大学出版社, 2006]

3 Cai S H. Modern Steel Tube Confined Concrete Structures (Revised Ed) (in Chinese). Beijing: China Communications Press, 2007 [蔡绍怀. 现代 钢管混凝土结构(修订版). 北京: 人民交通出版社, 2007]

4 Han L H, Li W, Bjorhovde R. Developments and advanced applications of concrete-filled steel tubular (CFST) structures: Members. J Constr Steel Res, 2014, 100: 211-228

5 Han L H, Li W, Wang W D, et al. Advanced Composite and Mixed Structures: Testing, Theory and Design Approach (in Chinese). 2nd ed. Beijing: Science Press, 2017 [韩林海, 李威, 王文达, 等. 现代组合结构和混合结构——试验、理论和方法. 第二版. 北京: 科学出版社, 2017]

6 Association for Steel-Concrete Composite Structures (ASCCS). Concrete Filled Steel Tubes-A Comparison of International Codes and Practices. ASCCS Seminar Report, Innsbruck, Austria, 1997

7 Shams M, Saadeghvaziri M A. State of the art of concrete-filled steel tubular columns. ACI Struct J, 1997, 94: 558-571

8 Shanmugam N E, Lakshmi B. State of the art report on steel-concrete composite columns. J Constr Steel Res, 2001, 57: 1041-1080

9 Gourley B C, Tort C, Denavit M D, et al. A Synopsis of Studies of the Monotonic and Cyclic Behavior of Concrete-Filled Steel Tube Members, Connections, and Frames. Report No. NSEL-008, NSEL Report Series, Department of Civil and Environmental Engineering, University of Illinois at Urbana-Champaign, 2008

10 GB 50923-2013. Technical Code for Concrete-Filled Steel Tube Arch Bridges (in Chinese). Beijing: China Planning Press, 2013 [中华人民共和国 国家标准GB 50923-2013. 钢管混凝土拱桥技术规范. 北京: 中国计划出版社, 2013] 
11 GB 50936-2014. Technical Code for Concrete Filled Steel Tubular Structures (in Chinese). Beijing: China Architecture \& Building Press, 2014 [中 华人民共和国国家标准GB 50936-2014. 钢管混凝土结构技术规范. 北京: 中国建筑工业出版社, 2014]

12 CECS 28:2012. Technical Specification for Concrete-Filled Steel Tubular Structures (in Chinese). Beijing: China Planning Press, 2012 [中国工程 建设标准化协会标准CECS 28:2012. 钢管混凝土结构技术规程. 北京: 中国计划出版社, 2012]

13 CECS 159:2004. Technical Specification for Structures with Concrete-Filled Rectangular Steel Tube Members (in Chinese). Beijing: China Planning Press, 2004 [中国工程建设标准化协会标准CECS 159:2004. 矩形钢管混凝土结构技术规程. 北京: 中国计划出版社, 2004]

14 DL/T 5085-1999. Code for Design of Steel-Concrete Composite Structure (in Chinese). Beijing: China Electric Power Press, 1999 [中华人民共和 国电力行业标准DL/T 5085-1999. 钢-混凝土组合结构设计规程. 北京: 中国电力出版社, 1999]

15 DBJ/T13-51-2010. Technical Specification for Concrete-Filled Steel Tubular Structures (in Chinese). Fuzhou: Fujian Provincial Department of Housing and Urban-Rural Development, 2010 [福建省工程建设标准DBJ/T13-51-2010. 钢管混凝土结构技术规程. 福州: 福建省住房和城乡建 设厅, 2010]

16 Architectural Institute of Japan. Recommendations for Design and Construction of Concrete Filled Steel Tubular Structures. Tokyo: Architectural Institute of Japan, 2008

17 ANSI/AISC 360-16. Specification for Structural Steel Buildings. Chicago: American Institute of Steel Construction (AISC), 2016

18 EN 1994-1-1. Eurocode 4: Design of Composite Steel and Concrete Structures-Part 1-1: General Rules and Rules for Buildings. Brussels: European Committee for Standardization, 2004

19 Dai X H, Lam D, Jamaluddin N, et al. Numerical analysis of slender elliptical concrete filled columns under axial compression. Thin-Walled Struct, 2014, 77: 26-35

20 Han L H, Yao G H, Zhao X L. Tests and calculations for hollow structural steel (HSS) stub columns filled with self-consolidating concrete (SCC). J Constr Steel Res, 2005, 61: 1241-1269

21 Du Y, Chen Z, Wang Y B, et al. Ultimate resistance behavior of rectangular concrete-filled tubular beam-columns made of high-strength steel. J Constr Steel Res, 2017, 133: 418-433

22 Tao Z, Yu Q. New Composite Structural Columns: Testing, Theory and Design Approach (in Chinese). Beijing: Science Press, 2006 [陶忠, 于清. 新型组合结构柱——试验、理论与方法. 北京: 科学出版社, 2006]

23 Han L H, Mou T M, Wang F C, et al. Design theory of CFST (concrete-filled steel tubular) mixed structures and its applications in bridge engineering (in Chinese). China Civil Eng J, 2020, 53: 1-24 [韩林海, 牟廷敏, 王法承, 等. 钢管混凝土混合结构设计原理及其在桥梁工程中的 应用. 土木工程学报, 2020, 53: 1-24]

24 Han L H, Hou C, Hua Y X. Concrete-filled steel tubes subjected to axial compression: Life-cycle based performance. J Constr Steel Res, 2020, 170: 106063

25 Han L H, Yao G H, Tao Z. Performance of concrete-filled thin-walled steel tubes under pure torsion. Thin-Walled Struct, 2007, 45: 24-36

26 Han L H. Tests on stub columns of concrete-filled RHS sections. J Constr Steel Res, 2002, 58: 353-372

27 Yang Y F, Han L H. Experimental behaviour of recycled aggregate concrete filled steel tubular columns. J Constr Steel Res, 2006, 62: 1310-1324

28 Han L H. Flexural behaviour of concrete-filled steel tubes. J Constr Steel Res, 2004, 60: 313-337

29 Yang H, Han L H, Wang Y C. Effects of heating and loading histories on post-fire cooling behaviour of concrete-filled steel tubular columns. J Constr Steel Res, 2008, 64: 556-570

30 Li G C, Yang Z J, Lang Y, et al. Behavior of high strength concrete filled square steel tubular columns with inner CFRP circular tube under bi-axial eccentric loading. Adv Steel Constr, 2013, 9: 231-246

31 Zhu A, Zhang X, Zhu H, et al. Experimental study of concrete filled cold-formed steel tubular stub columns. J Constr Steel Res, 2017, 134: 17-27

32 Cai J, Pan J, Wu Y. Mechanical behavior of steel-reinforced concrete-filled steel tubular (SRCFST) columns under uniaxial compressive loading. Thin-Walled Struct, 2015, 97: 1-10

33 Zhou T, Ren Z, Jiang B, et al. Calculating method study and parameter analysis of slender LCFST columns under axial loading. Int J Steel Struct, 2019, 19: 1645-1661

34 Li C, Hao H, Bi K. Numerical study on the seismic performance of precast segmental concrete columns under cyclic loading. Eng Struct, 2017, 148: $373-386$

35 Mashiri F R, Uy B, Tao Z, et al. Concrete-filled VHS-to-steel fabricated section stub columns subjected to axial compression. J Constr Steel Res, 2014, 95: 141-161

36 Tam V W Y, Wang Z B, Tao Z. Behaviour of recycled aggregate concrete filled stainless steel stub columns. Mater Struct, 2014, 47: 293-310

37 Li Y L, Zhao X L, Raman S R K, et al. Tests on seawater and sea sand concrete-filled CFRP, BFRP and stainless steel tubular stub columns. ThinWalled Struct, 2016, 108: 163-184

38 Javed M F, Sulong N H R, Memon S A, et al. FE modelling of the flexural behaviour of square and rectangular steel tubes filled with normal and high strength concrete. Thin-Walled Struct, 2017, 119: 470-481 
39 Aslani F, Uy B, Hur J, et al. Behaviour and design of hollow and concrete-filled spiral welded steel tube columns subjected to axial compression. J Constr Steel Res, 2017, 128: 261-288

40 Chen B, Sheng Y, Fam A, et al. Torsional behavior of a new dumbbell-shaped concrete-filled steel tubes. Thin-Walled Struct, 2017, 110: 35-46

41 Wang Z B, Yu Q, Tao Z. Behaviour of CFRP externally-reinforced circular CFST members under combined tension and bending. J Constr Steel Res, 2015, 106: 122-137

42 Zhang X G, Liu G, Gao Y, et al. Research on the bearing capacity of hollow concrete-filled steel exterior wall composite bridge tower column (in Chinese). China Civil Eng J, 2018, 51: 90-98 [张喜刚, 刘高, 高原, 等. 中空型外壁钢板-混凝土组合桥塔塔柱承载力研究. 土木工程学报, 2018, 51: 90-98]

43 Gao F, Zhu H P, Zhang D H, et al. Experimental investigation on flexural behavior of concrete-filled pentagonal flange beam under concentrated loading. Thin-Walled Struct, 2014, 84: 214-225

44 Bambach M R. Design of hollow and concrete filled steel and stainless steel tubular columns for transverse impact loads. Thin-Walled Struct, 2011, 49: 1251-1260

45 Zhu A Z, Xu W, Gao K, et al. Lateral impact response of rectangular hollow and partially concrete-filled steel tubular columns. Thin-Walled Struct, 2018, 130: 114-131

46 Wang J, Zhang N, Guo S. Experimental and numerical analysis of blind bolted moment joints to CFTST columns. Thin-Walled Struct, 2016, 109: 185-201

47 Kang L, Leon R T, Lu X. Shear strength analyses of internal diaphragm connections to CFT columns. Steel Compos Struct, 2015, 18: 1083-1101

48 Nie J, Qin K, Cai C S. Seismic behavior of connections composed of CFSSTCs and steel-concrete composite beams-Finite element analysis. J Constr Steel Res, 2008, 64: 680-688

49 Tang X L, Cai J, Chen Q J, et al. Seismic behaviour of through-beam connection between square CFST columns and RC beams. J Constr Steel Res, 2016, 122: 151-166

50 Nie J G, Ma X W, Tao M X, et al. Effective stiffness of composite shear wall with double plates and filled concrete. J Constr Steel Res, 2014, 99: 140-148

51 Chen Z H, Jiang Y T, Zhang X M, et al. Parametric analysis and calculation method for bending and shear capacities of innovative composite shear walls. Adv Struct Eng, 2017, 20: 1046-1058

52 Zhou L, Su Y. Experimental study on seismic behavior of SRC deep beam-to-CFST column frames. J Constr Steel Res, 2019, 155: 157-175

53 Wang K, Lu X F, Yuan S F, et al. Analysis on hysteretic behavior of composite frames with concrete-encased CFST columns. J Constr Steel Res, 2017, 135: 176-186

54 Wang J, Li B, Li J. Experimental and analytical investigation of semi-rigid CFST frames with external SCWPs. J Constr Steel Res, 2017, 128: 289304

55 Wang J X, Wang W D. Analysis of planar frame with steel beam-concrete-filled steel tubular columns after exposure to complete fire process under different fire cases (in Chinese). J Build Struct, 2014, 35: 102-109 [王景玄, 王文达. 不同火灾工况下钢梁-钢管混凝土柱平面框架受火全过程 分析. 建筑结构学报, 2014, 35: 102-109]

56 Wang Y, Nie J, Cai C S. Numerical modeling on concrete structures and steel-concrete composite frame structures. Compos Pt B-Eng, 2013, 51: $58-67$

57 Jiang H, Fu B, Liu L, et al. Study on seismic performance of a super-tall steel-concrete hybrid structure. Struct Des Tall Spec Build, 2014, 23: 334349

$58 \mathrm{Lu} \mathrm{X}, \mathrm{Lu}$ X, Guan H, et al. Earthquake-induced collapse simulation of a super-tall mega-braced frame-core tube building. J Constr Steel Res, 2013, 82: 59-71

59 Sun F F, Ran M M, Zhou J, et al. Mechanical behavior of huge crossover joint between two concrete-filled steel tubular columns (in Chinese). J Build Struct, 2017, 38: 69-76 [孙飞飞, 再明明, 周健, 等. 钢管混凝土柱巨型交叉节点受力性能研究. 建筑结构学报, 2017, 38: 69-76]

60 Liu Q X, Wang Q W, Zhang J J, et al. Performance-based seismic design on the ultra high-rise steel structure of tower A of Kashi International Duty-free Plaza (in Chinese). Build Struct, 2014, 44: 7-12 [刘琼祥, 王启文, 张建军, 等. 喀什国际免税广场A塔超高层钢结构性能化抗震设计. 建筑结构, 2014, 44: 7-12]

61 Lu J L, Yang L L, Gong M F, et al. Elastic-plastic time-history analysis of the main building of Guohai Plaza in Taiyuan under rare earthquake (in Chinese). Build Struct, 2014, 44: 42-46, 74 [路江龙, 杨律否, 龚敏锋, 等. 太原国海广场主楼罕遇地震弹塑性时程分析. 建筑结构, 2014, 44: 42-46, 74]

62 Yuan K, Li Y M, Liu L P, et al. Deformation and energy-based seismic performance analysis of super tall buildings in low seismic intensity region (in Chinese). Build Struct, 2014, 44: 13-18 [袁康, 李英民, 刘立平, 等. 基于变形和能量的低烈度区超高层建筑抗震性能分析. 建筑结构, 2014, 44: 13-18]

63 Guo Y L, Wang Y H. Analytical studies on design method and application of multi-column out-plane buckling in open-space region of the 
Guangzhou New TV Tower (in Chinese). J Build Struct, 2010, 31: 87-93 [郭彦林, 王永海. 广州新电视塔透空区外筒群柱面外稳定承载力与设 计方法研究. 建筑结构学报, 2010, 31: 87-93]

64 Zhang J M, Xiao R C. Ultimate bearing capacity analysis of the Wu Gorge Bridge over Yangtze River (in Chinese). J Highway Transport Res Dev, 2004, 21: 37-40 [张建民, 肖汝诚. 巫峡长江大桥极限承载能力分析. 公路交通科技, 2004, 21: 37-40]

65 Zhang J M, Zheng J L, Qin R. Double nonlinear stability analysis for the Yonghe Bridge (in Chinese). J Highway Transport Res Dev, 2002, 19: 5862 [张建民, 郑皆连, 秦荣. 南宁永和大桥双重非线性稳定分析. 公路交通科技, 2002, 19: 58-62]

66 Fan H, Wang L J, Wen S Q, et al. Dynamic elastic-plastic analysis on the main stadium of Wuhan Optical Valley International Tennis Center (in Chinese). Build Struct, 2016, 46: 50-58 [范浩, 王丽君, 温四清, 等. 武汉光谷国际网球中心主场馆动力弹塑性分析. 建筑结构, 2016, 46: 50$58]$

67 Li Z, Wang W D, Yu Q. Calculation and practice for fire resistance of concrete-filled steel tubular columns in underground engineering (in Chinese). Railway Eng, 2009, (9): 60-63 [李竹, 王文达, 于清. 地下工程中钢管混凝土柱的抗火计算与实践. 铁道建筑, 2009, (9): 60-63]

68 GB 50016-2006/2014. Code for Fire Protection Design of Buildings (in Chinese). Beijing: China Planning Press, 2006, 2014 [中华人民共和国国 家标准GB 50016-2006/2014. 建筑设计防火规范. 北京: 中国计划出版社, 2006, 2014]

69 GB 51249-2017. Code for Fire Safety of Steel Structures in Buildings (in Chinese). Beijing: China Planning Press, 2017 [中华人民共和国国家标 准GB 51249-2017. 建筑钢结构防火技术规范. 北京: 中国计划出版社, 2017]

70 CECS 200:2006. Technical Code for Fire Safety of Steel Structure in Buildings (in Chinese). Beijing: China Planning Press, 2006 [中国工程建设 标准化协会标准CECS 200:2006. 建筑钢结构防火技术规范. 北京: 中国计划出版社, 2006]

71 JGJ/T 249-2011. Technical Specification for Steel Arch Structure (in Chinese). Beijing: China Architecture \& Building Press, 2011 [中华人民共和 国行业标准JGJ/T 249-2011. 拱形钢结构技术规程. 北京: 中国建筑工业出版社, 2011]

72 JTG/T D65-06-2015. Specifications for Design of Highway Concrete-Filled Steel Tubular Arch Bridges (in Chinese). Beijing: China Communications Press Co., Ltd., 2015 [中华人民共和国行业推荐性标准JTG/T D65-06-2015. 公路钢管混凝土拱桥设计规范. 北京: 人民交通 出版社股份有限公司, 2015]

73 T/CECS 546-2018. Technical Standard for Structures with Concrete-Filled Multicellular Steel Tube Walls (in Chinese). Beijing: China Planning Press, 2018 [中国工程建设标准化协会标准T/CECS 546-2018. 钢管混凝土束结构技术标准. 北京: 中国计划出版社, 2018]

74 T/CECS 625-2019. Technical Specification for Recycled Aggregate Concrete-Filled Steel Tubular Structures (in Chinese). Beijing: China Architecture \& Building Press, 2019 [中国工程建设标准化协会标准T/CECS 625-2019. 钢管再生混凝土结构技术规程. 北京: 中国建筑工业 出版社, 2019] 


\title{
Life-cycle based analytical theory of concrete-filled steel tubular structures and its applications
}

\author{
Lin-Hai Han ${ }^{1 *}$, Youfu Yang ${ }^{2}$, Hua Yang ${ }^{3} \&$ Wei $\mathrm{Li}^{1}$ \\ ${ }^{1}$ Department of Civil Engineering, Tsinghua University, Beijing 100084, China; \\ ${ }^{2}$ State Key Laboratory of Costal and Offshore Engineering, Dalian University of Technology, Dalian 116024, China; \\ ${ }^{3}$ School of Civil Engineering, Harbin Institute of Technology, Harbin 150090, China \\ * Corresponding author, E-mail: 1hhan@tsinghua.edu.cn
}

Concrete-filled steel tube (CFST) is a composite structure consisting of a steel tube filled with concrete. The steel tube and its concrete core work together to carry external loads applied to the structure. Due to the interaction between the two kinds of materials, i.e., the confinement effect of steel tube on the core concrete, CFST structures can effectively delay/avoid the local buckling of the steel tube and the brittle failure of the concrete core. The superior structural properties of CFST include high strength and stiffness, good ductility, excellent seismic and fire performances, and easy construction. Therefore, CFST structures have been extensively used in large-scale constructions including industrial plants, subway stations, transmission towers, high-rise buildings, and bridges, etc. CFST structures have also offered substantial benefits on architectural appearance and engineering economy.

The confinement effect in CFST throughout its service-life essentially contributes to the structural advantages, whilst it has, at the same time, brought about enormous complexities in the corresponding load-resisting mechanisms. Firstly, an indepth understanding and accurate characterization of the confinement effect, and the development of some feasible constitutive models for the core concrete that facilitate the analysis and design of major infrastructures, becomes the key issue to be addressed. The salient loading factors in the service-life of CFST structures need to be considered in the constitutive models, such as long-term sustained load, low-cycle cyclic load and full-range fire. Secondly, the establishment of damage mechanism analysis and strength prediction models for CFST structures considering long-term sustained load, complex loading conditions, earthquake and fire becomes another key issue that needs to be resolved for the safety design of major CFST constructions.

Life-cycle based damage mechanism and analytical theory of concrete-filled steel tubular structures have been investigated and established based on nearly 20 years of persistent and systematic study. The design methods for the ultimate loading capacity of CFST structures were further proposed. The major research outcomes can be summarized in the following three aspects: (1) The interaction between the steel tube and its core concrete as well as the passive confinement mechanism in CFST were revealed. A general constitutive model for the confined core concrete under various stress/action conditions was established with the "confinement factor" as an essential variable. The considered actions include long-term sustained stress, cyclic load and elevated temperature. (2) Theoretical models were established to account for the damage mechanism of CFST structures under long-term sustained load and complex loading conditions. The ultimate strength of CFST members under various loading conditions was analysed, including compression, tension, bending, shear, torsion, and the combinations of different loads and load paths. The creep and shrinkage of concrete as well as the deformation of CFST under long-term sustained load were accurately simulated. (3) Theoretical models were established to simulate the damage mechanism of CFST under earthquake and full-range fire. The hysteretic properties and ductility of CFST members under low-cycle cyclic loading were revealed. The model for calculating the fire resistance of CFST columns was proposed, in which the coupling effects of loads and full-range fire were taken into account.

This paper briefly reviews the development of CFST structures and outlines the life-cycle based analytical theory for CFST structures. Finally, the potential development trends of the CFST structures are discussed.

concrete-filled steel tubular structures, life-cycle, confinement effect, confinement factor, constitutive model, analytical theory

doi: 10.1360/TB-2020-0618 